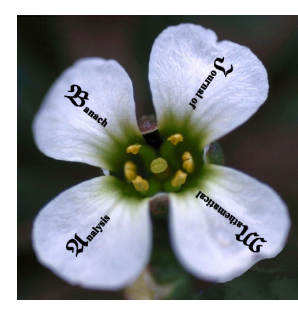

Banach J. Math. Anal. 8 (2014), no. 1, 109-117

Banach $\mathbf{J}_{\text {ournal of }} \mathbf{M}_{\text {athematical }} \mathbf{A}_{\text {nalysis }}$

ISSN: $1735-8787$ (electronic)

www.emis.de/journals/BJMA/

\title{
TWO CLASSES OF BOUNDS FOR THE $q$-GAMMA AND THE $q$-DIGAMMA FUNCTIONS IN TERMS OF THE $q$-ZETA FUNCTIONS
}

\author{
AHMED SALEM
}

Communicated by M. A. Ragusa

\begin{abstract}
In this paper, two infinite classes of inequalities for the $q$-gamma and the $q$-digamma functions are established by means of using the Taylor theorem. The bounds appeared in these inequalities are in terms of the Hurwitz and Riemann $q$-zeta functions.
\end{abstract}

\section{INTRODUCTION}

Recently, Batir [3] established inequalities below for $n=1,2,3$ and left the general case as a conjecture. Subsequently, $\mathrm{Yu}$ [20] completed the proof of this class for all $n \in \mathbb{N}$. These results provide bounds on the error of approximating $\log \Gamma(x+1)$ by means of a power series and state that: For $x>0$ and $n \in \mathbb{N}$, the following inequalities

$$
\begin{aligned}
\frac{(-1)^{n-1} x^{n+1}}{(n+1) !} \psi^{(n)}(\alpha(x)) & <(-1)^{n-1}\left(\log \Gamma(x+1)+\gamma x+\sum_{k=2}^{n}(-1)^{k-1} \zeta(k) \frac{x^{k}}{k}\right) \\
& <\frac{(-1)^{n-1} x^{n+1}}{(n+1) !} \psi^{(n)}(\beta(x))
\end{aligned}
$$

hold true for

$$
\alpha(x)=1+\frac{x}{n+2}
$$

Date: Received: 31 January 2013 ; Accepted: 6 May 2013.

2010 Mathematics Subject Classification. Primary 33D05; Secondary 26D07, 26A48.

Key words and phrases. Hurwitz and Riemann $q$-zeta functions, $q$-Gamma and $q$-digamma functions, inequality, Taylor theorem. 
and

$$
\beta(x)=x\left[(-1)^{n}(n+1)\left(\log (x+1)-\sum_{k=1}^{n} \frac{(-1)^{k-1} x^{k}}{k}\right)\right]^{\frac{-1}{n+1}}
$$

where $\Gamma, \psi$ and $\zeta$ are the gamma, digamma and Riemann zeta functions, respectively, and $\gamma$ is the Mascheroni-Eular constant (for their definitions, see [1]).

In the same manner, Chen and Srivastava [4] established bounds for the psi function in terms of the polygamma functions. Their results state that: For $x>0$ and $n \in \mathbb{N}$, the following inequalities

$$
\frac{(-1)^{n+1} \psi^{(n)}(\gamma(x))}{(n+1) !}<(-1)^{n}\left(\psi(x)-\log x+\sum_{i=1}^{n-1} \frac{\psi^{(i)}(x)}{i+1}\right)<\frac{(-1)^{n+1} \psi^{(n)}(\delta(x))}{(n+1) !}
$$

hold true for

$$
\gamma(x)=x+\frac{1}{n+2}
$$

and

$$
\delta(x)=\left\{(-1)^{n}(n+1)\left[\log \left(1+\frac{1}{x}\right)-\sum_{i=0}^{n-1} \frac{(-1)^{i}}{(i+1) x^{i+1}}\right]\right\}^{\frac{-1}{n+1}} .
$$

The $q$-analogue of the gamma function is defined as

$$
\Gamma_{q}(x)=(1-q)^{1-x} \prod_{k=0}^{\infty} \frac{1-q^{n+1}}{1-q^{n+x}}, \quad 0<q<1,
$$

and

$$
\Gamma_{q}(x)=(q-1)^{1-x} q^{\frac{x(x-1)}{2}} \prod_{k=0}^{\infty} \frac{1-q^{-(n+1)}}{1-q^{-(n+x)}}, \quad q>1 .
$$

The logarithmic derivative $\psi_{q}(x)$ of the $q$-gamma function is known as the $q$-psi or $q$-digamma function, that is, it is given by

$$
\psi_{q}(x)=\frac{d}{d x}\left(\log \Gamma_{q}(x)\right)=\frac{\Gamma_{q}^{\prime}(x)}{\Gamma_{q}(x)}
$$

which appeared in the work of Krattenthaler and Srivastava [13] when they studied the summations for the basic hypergeometric series. Some of its properties presented and proved in their work. Also, they proved that $\psi_{q}(x)$ tends to the digamma function $\psi(x)$ when letting $q \rightarrow 1$. For more details on the $q$-digamma function, see [14] which presented some properties and expansions associated with the $q$-digamma function. Some inequalities involve the $q$-gamma and $q$-digamma functions have been introduced in $[2,7,8,9,10,15,16,17]$ and the extensive references given therein.

From (1.7), for $0<q<1$ and for all real variable $x>0$, we get

$$
\psi_{q}(x)=-\log (1-q)+\log q \sum_{k=0}^{\infty} \frac{q^{x+k}}{1-q^{x+k}},
$$


and from (1.8), for $q>1$ and $x>0$, we obtain

$$
\psi_{q}(x)=-\log (q-1)+\log q\left[x-\frac{1}{2}-\sum_{k=0}^{\infty} \frac{q^{-x-k}}{1-q^{-x-k}}\right] .
$$

From the previous definitions, for a positive $x$ and $q \geq 1$, we get

$$
\begin{aligned}
& \Gamma_{q}(x)=q^{\frac{(x-1)(x-2)}{2}} \Gamma_{q^{-1}}(x), \\
& \psi_{q}(x)=\frac{2 x-3}{2} \log q+\psi_{q^{-1}}(x) .
\end{aligned}
$$

The Hurwitz zeta function [1]

$$
\zeta(s, z)=\sum_{k=0}^{\infty} \frac{1}{(k+z)^{s}}, \quad \Re(s)>1 ; z \notin \mathbb{Z}_{0}^{-} .
$$

has many different $q$-extensions. One of these $q$-extension that we need, has the form

$$
\zeta_{q}(s, t, z)=\sum_{k=0}^{\infty} \frac{q^{(z+k) t}}{[z+k]_{q}^{s}}, \quad \Re(t)>0 ; z \in \mathbb{C} / \mathbb{Z}_{0}^{-} .
$$

This form has been investigated at the first time by [18] when $t=s$ such that $\Re(s)>0$. Recently, Kawagoe et. al. [12] have studied this function for all $s, t \in \mathbb{C}$ such that $\Re(t)>0$. It is worth mentioning that, some integral representations for the Hurwitz q-zeta function have been studied in [19]. Also many properties and identities for this function, when $t=s-1$, have been investigated by [6]. In the case of $z=1$ the function (1.15) will reduce to the Riemann $q$-zeta function which has been investigated by many authors for instance [11] and [5]. Throughout this paper, we need the function (1.15) when $t=s$ and so we will use the abbreviated symbols $\zeta_{q}(s, z)$ for the Hurwitz $q$-zeta function and $\zeta_{q}(s)$ for the Riemann $q$-zeta function for all $s>0$ and $z>0$. Notice that the Hurwitz q-zeta function (1.15) tends to the classical Hurwitz zeta function (1.14) when letting $q \rightarrow 1$ which is related to polygamma functions by the identity

$$
\psi^{(n)}(x)=(-1)^{n+1} n ! \zeta(n+1, x), \quad n \in \mathbb{N}_{0} ; x>0 .
$$

The main objective of this paper is to extend and generalize the classes of inequalities (1.1)-(1.3) and (1.4)-(1.5) to the $q$-gamma and the $q$-digamma functions for all positive real numbers $x, q$ by means of finding new representations for them by using Taylor theorem.

\section{Useful Lemmas}

We begin this section with the following useful lemmas which are needed to complete the proof of the main theorem.

Lemma 2.1. Suppose that $x$ and $q$ are positive reals such that $0<q<1$. Then the logarithm of the q-gamma function can be represented as

$$
\log \Gamma_{q}(x+1)=-x \log (1-q)-\sum_{k=1}^{\infty} \log \left(1+\frac{q^{k}[x]_{q}}{[k]_{q}}\right)
$$


and the q-digamma function can be represented as

$$
\psi_{q}(x)=\log [x]_{q}+\sum_{k=0}^{\infty}\left\{\log \left(1+\frac{q^{x+k}}{[x+k]_{q}}\right)+\frac{q^{x+k} \log q}{1-q^{x+k}}\right\}
$$

and also

$$
\begin{aligned}
\psi_{q}(x) & =\frac{\log q}{q-1} \log \left(1-q^{x}\right)-\log (1-q)+\frac{\log q}{q-1} \\
& \times \sum_{k=0}^{\infty}\left\{\log \left(1+\frac{q^{x+k}}{[x+k]_{q}}\right)-\frac{q^{x+k}}{[x+k]_{q}}\right\} .
\end{aligned}
$$

Proof. The representation (2.1) comes immediately by integrating (1.10) from 1 to $x+1$. The series expansion of logarithm function gives

$$
\log \left(1+\frac{q^{x+k}}{[x+k]_{q}}\right)=\log \left(1-q^{x+k+1}\right)-\log \left(1-q^{x+k}\right)=\sum_{r=1}^{\infty} \frac{q^{(x+k) r}\left(1-q^{r}\right)}{r} .
$$

Taking the summation from $k=0$ to $\infty$ with interchanging the sums yields

$$
\sum_{k=0}^{\infty} \log \left(1+\frac{q^{x+k}}{[x+k]_{q}}\right)=\sum_{r=1}^{\infty} \frac{q^{x r}\left(1-q^{r}\right)}{r} \sum_{k=0}^{\infty} q^{k r}=\sum_{r=1}^{\infty} \frac{q^{x r}}{r}=-\log \left(1-q^{x}\right) .
$$

Comparing this result with the formula (1.10) completes the proof.

Lemma 2.2. Suppose that $x$ and $t$ are positive reals, $0<q<1$ and $n \in \mathbb{N}$. Then there exists $\theta(t, x)$ such that $0<\theta(t, x)<x$ and

$$
\log \left(1+\frac{q^{t}[x]_{q}}{[t]_{q}}\right)=\sum_{i=0}^{n-1} \frac{(-1)^{i} q^{t(i+1)}[x]_{q}^{i+1}}{(i+1)[t]_{q}^{i+1}}+\frac{(-1)^{n} q^{t(n+1)}[x]_{q}^{n+1}}{(n+1)[t+\theta(t, x)]_{q}^{n+1}} .
$$

Proof. By Taylor's theorem, the logarithm function can be expanded as

$$
\log (1+z)=\sum_{i=0}^{n-1} \frac{(-1)^{i} z^{i+1}}{i+1}+\frac{(-1)^{n} z^{n+1}}{(n+1)(1+\theta)^{n+1}}, \quad n \in \mathbb{N}
$$

where $0<\theta<z$. Replacing $\theta$ by $q^{t}[\theta(t, x)]_{q} /[t]_{q}$ and $z$ by $q^{t}[x]_{q} /[t]_{q}$, leading to $0<[\theta(t, x)]_{q}<[x]_{q}$ and the relation (2.4) is held. Notice that $0<\theta(t, x)<x$ if and only if $0<[\theta(t, x)]_{q}<[x]_{q}$ and $[t]_{q}+q^{t}[\theta(t, x)]_{q}=[t+\theta(t, x)]_{q}$.

Lemma 2.3. Suppose that $x$ and $q$ are positive reals such that $0<q<1$ and $n$ is a positive integer. Then the function

$$
f(t, x)=(-1)^{n}\left(\log \left(1+\frac{q^{t}[x]_{q}}{[t]_{q}}\right)-\sum_{i=0}^{n-1} \frac{(-1)^{i} q^{t(i+1)}[x]_{q}^{i+1}}{(i+1)[t]_{q}^{i+1}}\right)
$$

is strictly positive and decreasing for all $t>0$.

Proof. On differentiating with respect to $t$ gives

$$
\frac{\partial}{\partial t} f(t, x)=(-1)^{n}\left(\frac{q^{t} \log q\left(1-q^{x}\right)}{\left(1-q^{t}\right)\left(1-q^{t+x}\right)}-\frac{\log q}{1-q^{t}} \sum_{i=0}^{n-1}(-1)^{i}\left(\frac{q^{t}[x]_{q}}{[t]_{q}}\right)^{i+1}\right) .
$$


Using geometric series formula yields

$$
\frac{\partial}{\partial t} f(t, x)=\frac{\log q}{1-q^{t+x}}\left(\frac{q^{t}[x]_{q}}{[t]_{q}}\right)^{n+1}<0
$$

which tells that the function $t \mapsto f(t, x)$ is decreasing on $(0, \infty)$ and since $\lim _{t \rightarrow \infty} f(t, x)=0$, then $f(t, x)>0$ for all $t>0$ and fixed $x$.

Lemma 2.4. Suppose that $t, x$ and $q$ are positive reals such that $0<q<1$ and $n$ is a positive integer. Then the function $\theta(t, x)$ mentioned in Lemma 2.2 can be represented as

$$
\theta(t, x)=\frac{\log \left(q^{-t}-\left(1-q^{x}\right)[(n+1) f(t, x)]^{\frac{-1}{n+1}}\right)}{\log q}
$$

where $f(t, x)$ defined as in (2.5), and has the properties:

(1) $0<\theta(t, x)<x$ for all $t>0$.

(2) The function $t \mapsto \theta(t, x)$ is increasing for all $t>0$.

(3) $\lim _{t \rightarrow \infty} \theta(t, x)=\frac{\log \left(n+1+q^{x}\right)-\log (n+2)}{\log q} \quad$ for all $x>0$ and $n \in \mathbb{N}$.

Proof. The expression (2.7) and the first property can be easily obtained from the relations (2.4) and (2.5). On differentiating (2.7) with respect to $t$ followed by inserting (2.6) gives

$$
\frac{\partial}{\partial t} \theta(t, x)=-\frac{[(n+1) f(t, x)]^{\frac{n+2}{n+1}}-\frac{q^{t}[x]_{q}}{[t+x]_{q}}\left(\frac{q^{t}[x]_{q}}{[t]_{q}}\right)^{n+1}}{\left(1-\left(1-q^{x}\right) q^{t}[(n+1) f(t, x)]^{\frac{-1}{n+1}}\right)[(n+1) f(t, x)]^{\frac{n+2}{n+1}}} .
$$

Let the function

$$
h(t)=f(t, x)-\frac{1}{n+1}\left(\frac{q^{t}[x]_{q}}{[t]_{q}}\right)^{\frac{(n+1)^{2}}{n+2}}\left(\frac{q^{t}[x]_{q}}{[t+x]_{q}}\right)^{\frac{n+1}{n+2}} .
$$

Differentiation gives

$$
h^{\prime}(t)=\frac{-\log q}{\left(1-q^{t}\right)\left(1-q^{t+x}\right)}\left(\frac{q^{t}[x]_{q}}{[t]_{q}}\right)^{\frac{(n+1)^{2}}{n+2}}\left(\frac{q^{t}[x]_{q}}{[t+x]_{q}}\right)^{\frac{n+1}{n+2}} g(t)
$$

where

$$
g(t)=\frac{1}{n+2}\left(1-q^{t}\right)+\frac{n+1}{n+2}\left(1-q^{t+x}\right)-\left(1-q^{t}\right)^{\frac{1}{n+2}}\left(1-q^{t+x}\right)^{\frac{n+1}{n+2}} .
$$

Since the logarithm function is concave, then by using Jensen's inequality, we get $\log \left(\frac{\left(1-q^{t}\right)+(n+1)\left(1-q^{t+x}\right)}{n+2}\right)>\frac{\log \left(1-q^{t}\right)}{n+2}+\frac{(n+1) \log \left(1-q^{t+x}\right)}{n+2}, \quad t>0$ and so the function $g(t)>0$ for all $t>0$ which concludes that $h^{\prime}(t)>0$ for all $t>0$. Since $\lim _{t \rightarrow \infty} h(t)=0$, then $h(t)<0$ for all $t>0$ which yields $(\partial / \partial t) \theta(t, x)>0$ for all $t>0$. Therefore, the function $t \mapsto \theta(t, x)$ is increasing 
on $(0, \infty)$. We are now in the process of finding the value of the limit in the third statement. From (2.5), we have

$$
\begin{aligned}
& \lim _{t \rightarrow \infty}\left(q^{-t}-\left(1-q^{x}\right)[(n+1) f(t, x)]^{\frac{-1}{n+1}}\right) \\
& =\lim _{t \rightarrow \infty}\left(q^{-t}-\left(1-q^{x}\right)\left[(-1)^{n}(n+1) \sum_{i=n}^{\infty} \frac{(-1)^{i} q^{t(i+1)}[x]_{q}^{i+1}}{(i+1)[t]_{q}^{i+1}}\right]^{\frac{-1}{n+1}}\right) \\
& =\lim _{t \rightarrow \infty}\left(q^{-t}-\left(1-q^{x}\right)\left(\frac{q^{t}[x]_{q}}{[t]_{q}}\right)^{-1}\left[1-\frac{n+1}{n+2} \frac{q^{t}[x]_{q}}{[t]_{q}}+O\left(\left(\frac{q^{t}[x]_{q}}{[t]_{q}}\right)^{2}\right)\right]^{\frac{-1}{n+1}}\right) \\
& =\lim _{t \rightarrow \infty}\left(q^{-t}+\left(1-q^{-t}\right)\left[1+\frac{1}{n+2} \frac{q^{t}[x]_{q}}{[t]_{q}}+O\left(\left(\frac{q^{t}[x]_{q}}{[t]_{q}}\right)^{2}\right)\right]\right) \\
& =\lim _{t \rightarrow \infty}\left(1-\frac{1}{n+2} \frac{1-q^{x}}{1-q^{t}}+O\left(\frac{q^{t}[x]_{q}}{[t]_{q}}\right)\right)=\frac{n+1+q^{x}}{n+2} .
\end{aligned}
$$

Hence

$$
\theta(\infty, x)=\lim _{t \rightarrow \infty} \theta(t, x)=\frac{\log \left(n+1+q^{x}\right)-\log (n+2)}{\log q}
$$

In view of the Lemmas 2.1, 2.2 and 2.4 and the definition of the Hurwitz and Riemann q-zeta functions in the previous section, we can derive the following lemma

Lemma 2.5. Suppose that $x$ and $q$ are positive reals and $n$ is a positive integer such that $0<q<1$. Then the logarithm of the q-gamma function can be represented, for $0<\theta(k, x)<x$, as

$$
\begin{aligned}
\log \Gamma_{q}(x+1) & =-x \log (1-q)-\sum_{i=0}^{n-1} \frac{(-1)^{i}[x]_{q}^{i+1}}{i+1} \zeta_{q}(i+1) \\
& -\frac{(-1)^{n}[x]_{q}^{n+1}}{n+1} \sum_{k=1}^{\infty} \frac{q^{k(n+1)}}{[k+\theta(k, x)]_{q}^{n+1}}
\end{aligned}
$$

and the q-digamma function can be represented, for $0<\theta(k+x, 1)<1$, as

$$
\begin{aligned}
\psi_{q}(x) & =\frac{\log q}{q-1} \log \left(1-q^{x}\right)-\log (1-q)+\frac{\log q}{q-1} \sum_{i=1}^{n-1} \frac{(-1)^{i}}{i+1} \zeta_{q}(i+1, x) \\
& +\frac{\log q}{q-1} \frac{(-1)^{n}}{n+1} \sum_{k=0}^{\infty} \frac{q^{(x+k)(n+1)}}{[x+k+\theta(k+x, 1)]_{q}^{n+1}} .
\end{aligned}
$$

\section{The Main Results}

In this section, the main results of this paper will be derived as follow: 
Theorem 3.1. For all positive reals $x$ and $q$ and a positive integer $n$, the class of two-sided inequalities

$$
\begin{aligned}
& \frac{[x]_{\hat{q}}^{n+1} \hat{q}^{1-\alpha_{\hat{q}}(x)}}{n+1} \zeta_{\hat{q}}\left(n+1, \alpha_{\hat{q}}(x)\right)+\frac{x(x-1)}{2}(-1)^{n+1} H(q-1) \log q \\
< & (-1)^{n+1}\left(\log \Gamma_{q}(x+1)+x \log (1-\hat{q})+\sum_{i=0}^{n-1} \frac{(-)^{i}[x]_{\hat{q}}^{i+1} \zeta_{\hat{q}}(i+1)}{i+1}\right) \\
< & \frac{[x]_{\hat{q}}^{n+1} \hat{q}^{1-\beta_{\hat{q}}(x)}}{n+1} \zeta_{\hat{q}}\left(n+1, \beta_{\hat{q}}(x)\right)+\frac{x(x-1)}{2}(-1)^{n+1} H(q-1) \log q
\end{aligned}
$$

holds true for

and

$$
\alpha_{q}(x)=1+\frac{\log \left(n+1+q^{x}\right)-\log (n+2)}{\log q}
$$

$$
\begin{aligned}
& \beta_{q}(x) \\
& =\frac{\log \left(1-q\left(1-q^{x}\right)\left[(-1)^{n}(n+1)\left(\log \left(1+q[x]_{q}\right)-\sum_{i=0}^{n-1} \frac{(-1)^{i} q^{i+1}[x]_{q}^{i+1}}{i+1}\right)\right]^{\frac{-1}{n+1}}\right)}{\log q}
\end{aligned}
$$

where $H(\cdot)$ denotes the Heaviside step function and

$$
\hat{q}= \begin{cases}q & \text { if } \quad 0<q \leq 1 \\ q^{-1} & \text { if } \quad q \geq 1\end{cases}
$$

Proof. Lemma 2.4 tells that the function $\theta(k, x)$ is increasing for all $k \geq 0$ and since $0<q<1$, then we get

$$
q^{k+\theta(\infty, x)}<q^{k+\theta(k, x)}<q^{k+\theta(1, x)}
$$

which reveals that

$$
\frac{q^{k(n+1)}}{[k+\theta(\infty, x)]_{q}^{n+1}}<\frac{q^{k(n+1)}}{[k+\theta(k, x)]_{q}^{n+1}}<\frac{q^{k(n+1)}}{[k+\theta(1, x)]_{q}^{n+1}} .
$$

Taking the summation from $k=1$ to $\infty$ and comparing with (2.5), (2.8) and (1.15) to obtain the desired results when $0<q<1$. Notice that $\alpha_{q}(x)=$ $1+\theta(\infty, x)$ and $\beta_{q}(x)=1+\theta(1, x)$. It is not difficult to show that the theorem is true when $q \geq 1$ by using the equation (1.12).

Theorem 3.2. For all positive reals $x$ and $q$ and a positive integer $n$, the class of two-sided inequalities

$$
\begin{aligned}
& \frac{\log \hat{q}}{\hat{q}-1} \frac{\hat{q}^{x-\gamma_{\hat{q}}(x)}}{n+1} \zeta_{\hat{q}}\left(n+1, \gamma_{\hat{q}}(x)\right)+\frac{2 x-3}{2}(-1)^{n} H(q-1) \log q \\
< & (-1)^{n}\left(\psi_{q}(x)+\log (1-\hat{q})+\frac{\log \hat{q}}{1-\hat{q}} \log \left(1-\hat{q}^{x}\right)+\frac{\log \hat{q}}{1-\hat{q}} \sum_{i=1}^{n-1} \frac{(-)^{i} \zeta_{\hat{q}}(i+1, x)}{i+1}\right) \\
< & \frac{\log \hat{q}}{\hat{q}-1} \frac{\hat{q}^{x-\delta_{\hat{q}}(x)}}{n+1} \zeta_{\hat{q}}\left(n+1, \delta_{\hat{q}}(x)\right)+\frac{2 x-3}{2}(-1)^{n} H(q-1) \log q
\end{aligned}
$$


holds true for

$$
\gamma_{q}(x)=x+\frac{\log (n+1+q)-\log (n+2)}{\log q}
$$

and

$\delta_{q}(x)=\frac{\log \left(1-q^{x}(1-q)\left[(-1)^{n}(n+1)\left(\log \left(1+\frac{q^{x}}{[x]_{q}}\right)-\sum_{i=0}^{n-1} \frac{(-1)^{i} q^{x(i+1)}}{(i+1)[x]_{q}^{i+1}}\right)\right]^{\frac{-1}{n+1}}\right)}{\log q}$.

Proof. The proof of this theorem is similar as in the previous theorem.

Remark 3.3. It is easy to see that all results in Theorems 3.1 and 3.2 tend to the results from (1.1) to (1.6) when letting $q \rightarrow 1$ by using the relation (1.16) and l'Hospital's rule for limits. However, we show the following limit because it may make a confusion for some readers. In view of (3.1), we have

$$
\lim _{q \rightarrow 1}\left(x \log (1-q)+[x]_{q} \zeta_{q}(1)\right)=\lim _{q \rightarrow 1}\left(x \log (1-q)+\left(1-q^{x}\right) \sum_{k=1}^{\infty} \frac{q^{k}}{1-q^{k}}\right)
$$

Inserting (1.10) when $x=1$ gives

$$
\begin{aligned}
\lim _{q \rightarrow 1}\left(x \log (1-q)+[x]_{q} \zeta_{q}(1)\right) & =\lim _{q \rightarrow 1}\left(x \log (1-q)+\frac{1-q^{x}}{\log q}\left\{\psi_{q}(1)+\log (1-q)\right\}\right) \\
& =\lim _{q \rightarrow 1}\left(\frac{1-q^{x}}{\log q} \psi_{q}(1)+\frac{x \log q+1-q^{x}}{\log q} \log (1-q)\right) \\
& =-x \psi(1)-\lim _{q \rightarrow 1} \sum_{k=1}^{\infty} \frac{q^{k}\left(x \log q+1-q^{x}\right)}{k \log q} \\
& =-x \psi(1)=x \gamma
\end{aligned}
$$

which gives the same results in (1.1).

\section{REFERENCES}

1. M. Abramowitz and C.A. Stegun, Handbook of mathematical functions with formulas, graphs, and mathematical tables, National Bureau of Standards Applied Mathematics Series, 55 For sale by the Superintendent of Documents, U.S. Government Printing Office, Washington, D.C. 1964.

2. H. Alzer and A.Z. Grinshpan, Inequalities for the gamma and q-gamma functions, J. Approx. Theory 144 (2007), 67-83.

3. N. Batir, On some properties of the gamma function, Expo. Math. 26 (2008), 187-196.

4. C. Chen and H.M. Srivastava, A class of two-sided inequalities involving the psi and polygamma functions, Integral Transforms and Spec. Funct. 21 (2010), no. 7, 523-528.

5. I. Cherednik, On q-analogues of Riemann's zeta function, Selecta Math. (N.S.) 7 (2001), 447-491.

6. J. Choi, P.J. Anderson and H.M. Srivastava, Carlitz's q-Bernoulli and q-Euler numbers and polynomials and a class of generalized q-Hurwitz zeta functions, Appl. Math. Comput. 215 (2009), 1185-1208.

7. N. Elezović, C. Giordano and J. Pečarić, Convexity and q-gamma function, Rend. Circ. Mat. Palermo (2) 48 (1999), no. 2, 285-298. 
8. P. Gao, Some Monotonicity Properties of Gamma and q-Gamma Functions, ISRN Math. Anal. 2011 (2011), 1-15.

9. A.Z. Grinshpan and M.E.H. Ismail, Completely monotonic functions involving the gamma and q-gamma functions, Proc. Amer. Math. Soc. 134 (2006), 1153-1160.

10. M.E.H. Ismail and M.E. Muldoon, Inequalities and monotonicity properties for gamma and q-gamma functions, Approximation and computation (West Lafayette, IN, 1993), 309-323, Internat. Ser. Numer. Math., 119, Birkhäuser Boston, Boston, MA, 1994.

11. M. Kaneko, N. Kurokawa and M. Wakayama, A variation of Eulers approach to values of the Riemann zeta function, Kyushu J. Math. 57 (2003), 175-192.

12. K. Kawagoe, M. Wakayama and Y. Yamasaki, q-Analogues of the Riemann zeta, the Dirichlet L-functions, and a crystal zeta function, Forum Math. 20 (2008), no. 1, 1-26.

13. C. Krattenthaler and H.M. Srivastava, Summations for basic hypergeometric series involving a q-analogue of the digamma function, Comput. Math. Appl. 32 (1996), no. 3, 73-91.

14. A. Salem, Some Properties and Expansions Associated with the q-Digamma Function, Quaestiones mathematicae 36 (2013), no.1, 67-77.

15. A. Salem, A completely monotonic function involving q-gamma and q-digamma functions, J. Approx. Theory 164 (2012), no. 7, 971-980.

16. A. Salem, An infinite class of completely monotonic functions involving the q-gamma function, J. Math. Anal. Appl. 406 (2013), no. 2, 392-399.

17. A. Salem Complete monotonicity properties of functions involving q-gamma and q-digamma functions, Math. Inequal. Appl. (To appear)

18. K. Ueno and M. Nishizawa, Quantum groups and zeta-functions, Quantum groups (Karpacz, 1994), 115-126, PWN, Warsaw, 1995.

19. M. Wakayama and Y. Yamasaki, Integral representations of q-analogues of the Hurwitz zeta function, Monatsh. Math. 149 (2006), no. 2, 141-154.

20. Y. Yu, A remark on class of double inequalities of Batir, Expo. Math. 27 (2009), no. 2, $171-174$.

Department of Basic Science, Faculty of Information Systems \& Computer Science, October 6 University, Six of October City, Giza, Egypt.

E-mail address: ahmedsalem74@hotmail.com 